\title{
Biological functions of p53 isoforms through evolution: lessons from animal and cellular models
}

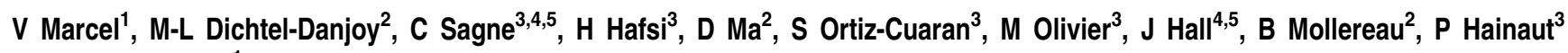 \\ and J-C Bourdon ${ }^{\star, 1}$
}

The TP53 tumour-suppressor gene is expressed as several protein isoforms generated by different mechanisms, including use of alternative promoters, splicing sites and translational initiation sites, that are conserved through evolution and within the TP53 homologues, TP63 and TP73. Although first described in the eighties, the importance of p53 isoforms in regulating the suppressive functions of p53 has only become evident in the last 10 years, by analogy with observations that p63 and p73 isoforms appeared indispensable to fully understand the biological functions of TP63 and TP73. This review summarizes recent advances in the field of ' $p 53$ isoforms', including new data on p63 and p73 isoforms. Details of the alternative mechanisms that produce p53 isoforms and cis- and trans-regulators identified are provided. The main focus is on their biological functions (apoptosis, cell cycle, aging and so on) in cellular and animal models, including mouse, zebrafish and Drosophila. Finally, the deregulation of p53 isoform expression in human cancers is reviewed. Based on these latest results, several developments are expected in the future: the identification of drugs modulating p53 isoform expression; the generation of animal models and the evaluation of the use of $\mathrm{p} 53$ isoform as biomarkers in human cancers.

Cell Death and Differentiation (2011) 18, 1815-1824; doi:10.1038/cdd.2011.120; published online 23 September 2011

Thirty years of research have shown that the p53 tumoursuppressor protein, encoded by TP53 gene (OMIM 191170), integrates endogenous and exogenous signals to modulate cell fate to stress and cellular environments. ${ }^{1}$ In addition, it has emerged that p53 is more than just a 'stress response' factor as it regulates embryo implementation. ${ }^{2}$ The ability of p53 to integrate signals implies the existence of multiple and subtle levels of regulation. Over the years, transcriptional, translational and post-translational regulatory mechanisms have been uncovered. . $^{3,4}$ This biochemical diversity echoes the genetic diversity of the TP53 locus, which contains multiple genetic polymorphisms defining over 100 distinct TP53 haplotypes. ${ }^{5}$ Recently, an additional layer of regulatory mechanism has emerged through identification of p53 isoforms, which are physiological proteins expressed in normal cells from the TP53 gene owing to the use of alternative promoters, splicing sites and/or translational initiation sites. ${ }^{6,7}$

The p53 isoforms were first identified in early studies investigating p53 expression patterns. In 1984, Matlashewski et al. cloned an N-terminal variant of the human p53 mRNA, whereas in 1985, Rotter and co-workers detected an alternatively spliced C-terminal variant of mouse $\mathrm{p53}$, latter isolated in human cells. ${ }^{8-10}$ However, the 'p53 isoform' field has only really emerged in the past 10 years, when it became clear that TP53 retained the elaborate patterns of isoform expression that characterizes its homologues, TP63 and TP73. The rapid accumulation of descriptive, functional and clinical data on p53 isoforms has led to the emergence of a research community, which held its First International Meeting at the International Agency for Research on Cancer in Lyon, France, in September 2010. ${ }^{11}$ This review provides a brief survey of the 'p53 isoforms' field at a time when it is emerging at the forefront of $p 53$ research.

\section{TP63 and TP73: the Isoform Paradigm}

The two p53-related proteins, p63 and p73, share strong structural, biochemical and biological homologies. ${ }^{12,13}$ In particular, they bind specifically to DNA onto conserved p53 response elements (p53REs) by using their DNA-binding domain. In the late nineties, the cloning of TP63 and TP73 revealed an elaborate pattern of mRNA expression resulting in several protein isoforms. ${ }^{14,15}$ Several N-terminal forms, produced by the use of alternative promoters and/or alternative splicing (i.e., TA forms, which contains the transactivation domain (TAD), versus $\Delta \mathrm{N}$ forms, produced from an

\footnotetext{
${ }^{1}$ Centre for Oncology and Molecular Medicine, INSERM-European Associated Laboratory, University of Dundee, Ninewells Hospital, Dundee, Scotland DD1 9SY, UK; ${ }^{2}$ Apoptosis and Neurogenetics Group, Ecole Normale Supérieure de Lyon, Laboratory of Molecular Biology of the Cell, CNRS UMR5239, University of Lyon, UMS3444 Biosciences Lyon Gerland, 46 allée d'Italie, Lyon 69007, France; ${ }^{3}$ Molecular Carcinogenesis Group, International Agency for Research on Cancer, Lyon Cedex 08, France; ${ }^{4}$ INSERM U612, Bât. 110-112, Centre Universitaire, Orsay 91405, France and ${ }^{5}$ Institut Curie Centre de Recherche, Bât. 110-112, Centre Universitaire, Orsay 91405, France

${ }^{*}$ Corresponding author: J-C Bourdon, Centre for Oncology and Molecular Medicine, INSERM-European Associated Laboratory, University of Dundee (Mailbox 4), Ninewells Hospital, Medical School, Dundee, Scotland DD1 9SY, UK. Tel: + 44 (0) 138249 6401; Fax: + 44 (0) 138 249 6363; E-mail: j.bourdon@ dundee.ac.uk Keywords: tumour suppressor; p53; p63; p73; p53 family; isoforms

Abbreviations: MEFs, mouse embryonic fibroblasts; TAD, transactivation domain; p53REs, p53 response elements

Received 19.5.11; revised 04.8.11; accepted 12.8.11; Edited by G Melino; published online 23.9.11
} 
internal promoter resulting in the presence of a different TAD), were found combined with several C-terminal forms generated by alternative splicing (five for p63: $\alpha$ to $\varepsilon$; seven for p73: $\alpha$ to $\eta) .{ }^{13}$ Far from being of minor component, the $\Delta$ Np63 and $\triangle \mathrm{Np} 73$ forms are the major forms expressed in certain cell types. ${ }^{14,16}$ In mice, knockout of the entire TP63 or TP73 loci (targeting all isoforms) revealed the roles of p63 and p73 in epithelial differentiation and neuronal development, respectively, ${ }^{15,17}$ whereas no impact was observed in stem cell commitment. ${ }^{18}$ However, with the generation of isoformspecific knockout mice, a subtle interplay between the $\mathrm{N}$-terminal isoforms has recently emerged, with the dynamic expression of $\mathrm{N}$-terminal p63 or p73 isoforms appearing critical for maintaining the normal sequence of cell development (from stem to committed progenitors and then differentiated cells). ${ }^{19,20}$ Building on this idea, Aberdam and co-workers analysed the impact of $\Delta$ Np63 and TAp63 isoforms in cellular commitment. In murine embryonic stem cells, $\Delta \mathrm{Np63}$, but not TAp63, is highly expressed during epidermal commitment and is critical for the expression of the cytokeratins $\mathrm{K} 14$ and $\mathrm{K} 5$, two markers of keratinocyte proliferation, indicating that only $\Delta \mathrm{Np} 63$ is required for the commitment of ectodermal into epidermal cells. ${ }^{21,22}$ Mills et al. observed that $\Delta \mathrm{Np} 63 \alpha$ overexpression in mouse embryonic fibroblasts (MEFs) resulted in the bypass of Ras-mediated senescence and enhanced carcinoma development in mice, suggesting that $\Delta \mathrm{Np} 63 \alpha$ inhibits senescence and therefore acts as an oncogene. ${ }^{23}$ By contrast, overexpression of TAp63 forms in $\mathrm{p} 53^{-1-}$ MEFs increased senescence and reduced tumour development in vivo, consistent with a p53-independent effect. ${ }^{24}$ Studying mice deficient for specific $\mathrm{p} 73$ protein isoforms, Mak, Melino and co-workers revealed that, like p63, the different p73 isoforms had dual cellular roles. In particular, $\mathrm{TAp} 73^{-/-}$mice are spontaneously tumour-prone whereas $\Delta \mathrm{Np} 3^{-1-}$ cells show impaired tumour formation in nude mice. ${ }^{25,26}$ In addition to these cancer-related effects, knockout of either TAp73 or $\triangle \mathrm{Np73}$ isoforms resulted in isoformspecific defects in neurogenesis or neurodegeneration, respectively. Overall, these studies show that each $\mathrm{N}$-terminal p63/p73 form has specific roles in regulating distinct cell differentiation pathways. Moreover, they demonstrate that the $\Delta \mathrm{N}$ forms, by preventing senescence and maintaining progenitor cell status, may act as oncogenes, whereas TA forms, through their capacity to switch cells into a post-mitotic state, may act as tumour suppressors.

\section{Generation of Human p53 Isoforms by Diverse Regulatory Mechanisms}

Like TP63 and TP73, the human TP53 gene encodes several p53 protein isoforms through conserved mechanisms. ${ }^{6,7}$ The main and most abundant p53 isoform is the canonical p53 protein, also termed TAp $53 \alpha$, as it contains an entire TAD and the longest $\mathrm{C}$-terminal domain (Figure 1). In addition to the TA forms, three $\Delta \mathrm{N}$ forms have been identified that differ by their translation initiation site. This is used to designate them as $\Delta 40 p 53, \Delta 133 p 53$ and $\Delta 160 p 53$. The four $\mathrm{N}$-terminal p53 forms can be combined with three different $\mathrm{C}$-terminal domains $(\alpha, \beta, \gamma)$. Recently, some cis- and trans-regulators have been identified as specific modulators of p53 isoform expression (Figure 1).

$\Delta 40$ p53 expression: one form, several mechanisms. Compared with $\mathrm{p} 53$, the human $\mathrm{N}$-terminal $\Delta 40 \mathrm{p} 53$ forms lack the first 39 amino acids corresponding to the main TAD (Figure 1c). Matlashewski et al. ${ }^{27}$ identified a p53 mRNA species retaining the entire intron-2 (p53|2), indicating that an alternative splicing event leading to the retention of the TP53 intron-2 can occur (Figure 1b). They later observed that stop codons in intron-2 of the p53I2 mRNA prevent p53 expression from the first AUG. ${ }^{28}$ However, p53I2-transfected cells were found to express a $45-\mathrm{kDa}$ protein, undetectable using antibodies recognizing the p53-TAD epitopes (DO1 or DO7), that corresponds to $\Delta 40 p 53$ initiated at a second AUG at codon-40, encompassed within a strong Kozak consensus. ${ }^{29}$ Alternative splicing of intron-2 can be regulated through G-quadruplex structures located in intron-3 of the p53 pre-mRNA. ${ }^{30}$ Using reporter assays and RNA-Gquadruplex ligands, it appears that G-quadruplex structures promote the correct splice-out of intron-2, leading to the fully spliced p53 (FSp53) mRNA encoding the full-length p53 protein; G-quadruplex disruption however favours the retention of intron-2 and thus p53I2 mRNA expression. This observation is the first clue that the TP53 sequence itself can modulate its own isoforms' expression through regulation of alternative splicing.

In addition to alternative splicing, $\Delta 40 p 53$ forms can be encoded from the FSp53 mRNA through an internal initiation of translation at codon-40. ${ }^{29}$ Two internal ribosomal entry sequences (IRES) have been identified that regulate the translation of either p53 or $\Delta 40$ p53 (Figure 1b). ${ }^{31,32}$ However, the relative contribution, in vivo, of each of these mechanisms, which are producing $\Delta 40 \mathrm{p} 53$, remains to be fully established.

Production of $\Delta 133 p 53$ and $\Delta 160 p 53$ forms from the internal P2 promoter. As for TP63 and TP73, TP53 contains an internal promoter that controls the expression of two N-terminal forms (Figure 1a). ${ }^{33}$ In addition to the proximal P1 promoter regulating p53 and $\Delta 40 \mathrm{p} 53$ expression, an internal P2 promoter located between intron-1 and exon-5 regulates the transcription of p53 mRNAs initiated in intron-4 (p53/4) (Figure 1b). ${ }^{33}$ This internal P2 promoter is different from the $\mathrm{P}^{\star}$ promoter in TP53 intron-1 identified by Reisman et al. ${ }^{34,35}$ that regulates the expression of an unrelated p53 transcript encoded by TP53 intron-1. Site-directed mutagenesis and siRNA methods revealed that translation of p5314 mRNAs can be initiated at two distinct codons, AUG133 and AUG160, leading to the expression of the $\Delta 133 p 53$ and $\Delta 160 p 53$ proteins, respectively, that lack the TAD and part of the DNA-binding domain (Figure 1c). ${ }^{36}$ Surprisingly, $\Delta 160 p 53$ forms are expressed in K562 cells, which are considered as 'p53-null' cells. The TP53 mutation in $\mathrm{K} 562$ cells results in a premature stop codon between AUG133 and AUG160, thus preventing the expression of the $\mathrm{TA}, \Delta 40$ and $\Delta 133$ forms without compromising the $\Delta 160 \mathrm{p} 53$ reading frame. Thus, it should be kept in mind that some cells or tumours considered as 'p53-null' because of the presence of frameshift or nonsense mutations, may retain the capacity to express one or several p53 isoforms. ${ }^{29,36}$ 
a

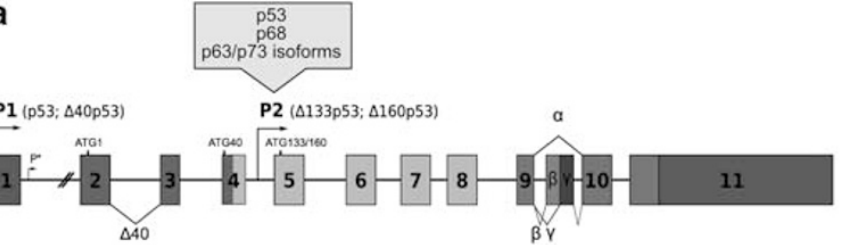

b

FSp53

\begin{tabular}{l|l}
\hline IRES-1] [RES-2 \\
\hline
\end{tabular}

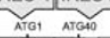

FSp53 $\beta$

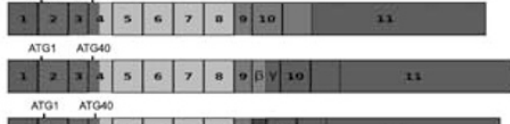

Detected at
endogenous
level

FSp53y

ATOS ATOAO

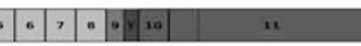

[8]

[33]

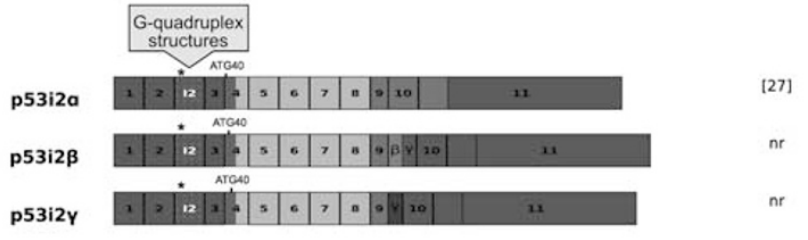

p53i2y

$1=|12|$

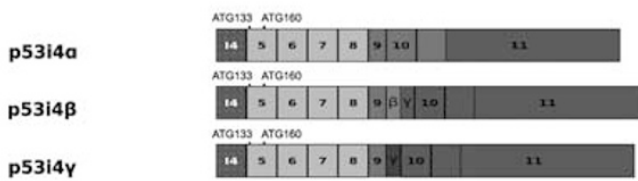

[33]

[33]

[33]

c

$p 53$
$p 53 \beta$
$p 53 y$
$\Delta 40 p 53 a$
$\Delta 40 p 53 \beta$
$\Delta 40 p 53 y$
$\Delta 133 p 53 a$
$\Delta 133 p 53 \beta$
$\Delta 133 p 53 y$

$\Delta 160 p 53 a$
$\Delta 160 p 53 \beta$
$\Delta 160 p 53 y$

TAD PXXP$$
\text { (I) (II) }
$$$$
\text { ATG } 1
$$

DBD
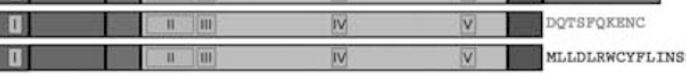

ATG 40

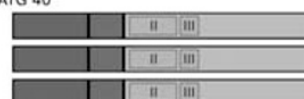

IV

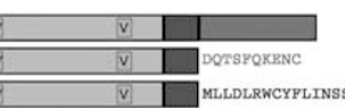

ATG 133

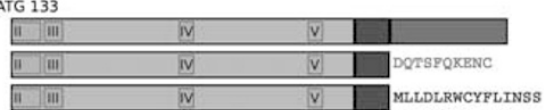

ATG 160

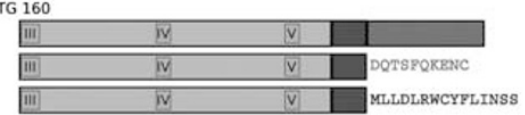

OD

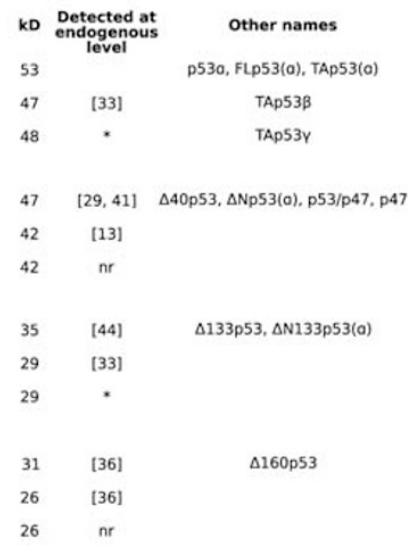

Figure 1 A schematic representation of human p53 isoforms. (a) The human TP53 gene structure. The TP53 gene, which consists of 11 exons (coloured boxes, coding exons; grey boxes, non-coding exons), expresses several p53 isoforms owing to usage of alternative promoters $(\ulcorner)$, splicing sites $(\wedge)$ or translational initiation sites $(I)$. (b) Human p53 mRNA variants. The proximal promoter P1, located upstream from exon-1, regulates the transcription of two transcripts: the fully spliced p53 mRNA (FSp53), which encodes both p53 (from ATG1) and $\Delta 40 \mathrm{p} 53$ forms (from ATG40), and the p5312 mRNA, retaining the entire intron-2 by alternative splicing, which generates $\Delta 40 \mathrm{p} 53$ forms from ATG40, owing to the presence of stop codons $\left({ }^{*}\right)$ in the reading frame starting from ATG1. The internal P2 promoter, described as encompassing the region from intron-1 to exon-5, produces p5314 mRNA, initiated in intron-4 and encoding the N-terminal $\Delta 133$ p53 (from ATG133) and $\Delta 160$ p53 forms (from ATG160). Three different C-terminal p53 forms have been described owing to alternative splicing of intron-9: the $\alpha$-forms resulting from the excision of the entire intron-9, and the $\beta$ - and $\gamma$-forms produced by retention of two small parts of intron-9. Some cis- and trans-regulators driving p53 isoform expression have been described (purple boxes). Endogenous expression of most of the p53 mRNA variants in human cells has been reported (references shown in parentheses). Grey box, non-coding sequence; NR, not yet reported. (c) Human p53 protein isoforms. The canonical p53 protein contains a TAD (blue), a proline-rich domain (PXXP, purple), a DNA-binding domain (DBD, orange) and an OD (green) that encompasses a nuclear localization domain (NLS, green) and five regions conserved through evolution (I-V in grey boxes). Compared with p53, the $\Delta 40 \mathrm{p} 53$ forms lack the first TAD, whereas the $\Delta 133 p 53$ and $\Delta 160 p 53$ isoforms lack the entire TAD and parts of the DBD. At the C-terminal, the $\alpha$-peptide corresponds to the OD that is replace by new residues, the $\beta$ - and $\gamma$-peptides (brown). On the right is indicated the theoretical molecular weight, the detection at endogenous levels (reference in brackets) as well as the different names of the isoforms used in the literature. The color reproduction of this figure is available at the Cell Death and Differentiation journal online

Experimental studies showed a p53-dependent regulation of $\Delta 133 p 53$ expression. ${ }^{37,38}$ The internal $P 2$ promoter contains p53REs located at the junction of exon-4/intron-4. Promoter deletion, site-directed mutagenesis and chromatin
IP experiments demonstrate that the direct binding of p53 onto the p53REs results in an increased expression of both $\Delta 133 p 53 \mathrm{mRNAs}$ and the $\Delta 133 p 53 \alpha$ protein. Some experimental evidence suggests that protein expression of 
$\Delta 133 p 53 \alpha$ can be induced through p53-independent mechanisms. In particular, the accumulation of the $\Delta 133 p 53 \alpha$ protein was observed in response to the knockdown of p68, a DEAD Box helicase involved in multiple transcriptional regulatory processes, and more recently in response to the expression of some $\mathrm{p} 63$ or p73 isoforms. ${ }^{39}$

The C-terminal spliced p53 forms, $\boldsymbol{\beta}$ and $\gamma$. The alternative splicing of intron-9 of human TP53 produces three different C-terminal p53 forms $\left(\alpha, \beta\right.$ and $\gamma$ ) (Figure 1a) ${ }^{8,33}$ Complete excision of intron- 9 results in the expression of the $\alpha$-forms corresponding to the classical p53 C-terminal domain (oligomerization domain, OD) (Figures $1 \mathrm{~b}$ and $\mathrm{c}$ ). On the other hand, partial retention of intron-9 generates the $\beta$ - or $\gamma$-forms, in which the OD is replaced by 10 or 15 new amino acids, respectively. However, the mechanisms that control the alternative splicing of intron-9 are unknown.

The classical p53 C-terminal domain contains the main post-translational modification sites regulating p53 stability, such as the lysines residues ubiquitinated by Mdm2, an E3ubiquitin ligase regulating p53 stability and activity. ${ }^{3}$ Their absence in $\beta$ - and $\gamma$-forms led to the investigation as to whether the stability of the $\mathrm{p} 53 \beta$ and $\mathrm{p} 53 \gamma$ proteins is regulated by the Mdm2-ubiquitin-proteasome pathway. To date, results remain controversial. For instance, whereas constitutively overexpressed FLAG-tagged p53 $\beta$ or $p 53 \gamma$ do not appear to interact with $\mathrm{Mdm} 2$ or to be degraded in a proteasomedependent manner, ${ }^{40}$ Bourdon and co-workers observed that the endogenous $\mathrm{p} 53 \beta$ protein can be degraded by the proteasome in an Mdm2-dependent manner.

\section{Biological Functions of Human p53 Isoforms}

Based on early studies detailed below, the $\mathrm{N}$-terminal isoforms lacking the TAD (i.e., $\Delta 40$ p53, $\Delta 133 p 53$ and $\Delta 160 p 53)$ were expected to only act as dominant-negative regulators of p53 activity. For instance, ectopic $\Delta 40 p 53 \alpha$ expression downregulated the p53-induced transactivation on reporter genes and counteracted p53-dependent growth suppression in colony formation assays. ${ }^{28,29}$ In addition, in human diploid fibroblast WI38 cells, expression of endogenous $\Delta 40 p 53 \alpha$ increased during the $\mathrm{G}_{1} / \mathrm{S}$ transition, in parallel with decreased expression of p21. ${ }^{29}$ Furthermore, as $\Delta 40 p 53 \alpha$ lacks the Mdm2-binding site, it escapes Mdm2mediated degradation and does not accumulate in response to DNA damage, its expression persisting at low but stable amounts in many cell types. ${ }^{29,41}$ These data support the notion that $\Delta 40 p 53 \alpha$ inhibits basal p53 activities during cellcycle progression. However, whether $\Delta 40 p 53 \alpha$ can exert $p 53-$ independent effects is still unknown. Hainaut and co-workers observed that $\Delta 40 p 53 \alpha$ contains an intact DNA-binding domain able to bind to p53REs in vitro. Thus, $\Delta 40 p 53 \alpha$ may also exert intrinsic regulatory effects by competing with $\mathrm{p} 53$ for p53RE binding and thereby modulating their accessibility for other transcription factors, regulating the cell fate outcome depending upon cell type and cell context. In addition, $\Delta 40 p 53 \alpha$ lacks the first TAD, which has been shown to be dispensable for p53 transcriptional activity, and retains the second TAD, which can regulate gene expression. ${ }^{42,43}$ Therefore, with our current knowledge, one should be cautious in considering $\Delta 40 p 53$ as a simple dominantnegative inhibitor of p53.

Compared with $\Delta 40 p 53 \alpha$, the available data have clearly revealed that $\Delta 133 p 53$ controls p53 activity. In reporter assays, $\Delta 133 p 53 \alpha$ can also behave as a dominant-negative inhibitor of p53. ${ }^{13,33} \Delta 133 p 53 \alpha$ does not bind to consensus p53REs in vitro, consistent with its partial lack of the DNAbinding domain, and thus can also behave as a dominant mutant p53 protein. ${ }^{38}$ Experimental studies suggested that, instead of being a strict dominant inhibitor, $\Delta 133 p 53 \alpha$ is instead a fine modulator of p53's suppressive activity as its expression determines cell fate in response to stress. The knockdown of $\Delta 133 p 53 \alpha$ expression promotes p53-mediated apoptosis and $\mathrm{G}_{1}$ cell-cycle arrest in response to doxorubicin treatment, without altering the p53-dependent $G_{2}$ cell-cycle arrest. $^{37}$ These effects may be due to the ability of $\Delta 133 p 53 \alpha$ to modulate gene expression in a promoter-dependent manner, as observed for p21WAF1, Mdm2 and Bcl-2.37 Interestingly, $\Delta 133 p 53$ silencing has also been associated with replicative, but not oncogene-induced, senescence in normal human fibroblasts through transcriptional regulation of p53-target genes, including p21 WAF1 and mir-34a.44 Overall, these results are consistent with an oncogenic capacity of $\Delta 133 p 53$.

The biological functions of the $\mathrm{C}$-terminal p53 isoforms (i.e., $\mathrm{p} 53 \beta$ and $\mathrm{p} 53 \gamma$ ) remain poorly described and controversial. Bourdon et al. ${ }^{33}$ showed that, in the absence of stress, endogenous $\mathrm{p} 53 \beta$ bound to the Bax and $p 21^{W A F 1}$ promoters, but only weakly to that of Mdm2. Moreover, in luciferase reporter assays in the absence of stress, the co-expression of p53 $\beta$ and p53 enhanced the p53 transcriptional activity on the p21 ${ }^{W A F 1}$ promoter but not on the Bax promoter, suggesting a promoter-dependent effect. These observations are consistent with the demonstration that $p 53 \beta$ cooperates with $p 53$ to accelerate senescence in human fibroblasts. ${ }^{44}$ By contrast, experimental studies failed to observe binding of FLAGtagged $\mathrm{p} 53 \beta$ or $\mathrm{p} 53 \gamma$ onto p53RE consensus and to show a role of FLAG-tagged p53 $\beta$ or p53 $\gamma$ in p53-dependent apoptosis or senescence in cells constitutively overexpressing FLAG-tagged $\mathrm{p} 53 \beta$ or $\mathrm{p} 53 \gamma$, and selected to grow in presence of neomycin. ${ }^{40}$ Thus, there is still debate on whether $\mathrm{p} 53 \beta$ or p $53 \gamma$ exert their activities in an autonomous manner or through an interaction with p53. Furthermore, there is no evidence of distinct biological activities for p53 $\beta$ or p53 $\gamma$.

Overall, current experimental data on the biological roles of p53 isoforms are fragmented. Given that p53 isoforms differ from each other in the three functional domains (TAD, DNAbinding and OD), their potential to modulate p53-dependent responses is expected to be diverse and cell type-dependent. Further insight into which of these functions are of physiological or pathological relevance may come from animal model studies.

\section{Animal Models: clues to the Physiological Significance of Isoforms}

The simplest animal model to study p53 isoforms, Drosophila melanogaster. The diversification of the p53 gene family into three members occurred in vertebrates. 
Thus, invertebrates such as Drosophila contain a single p53related gene, which encodes three protein isoforms (Figure 2): Dp53, corresponding to the human full-length p53; D $\Delta$ Np53, a general counterpart of the human $\mathrm{N}$-terminal p53 forms as it is encoded by an mRNA transcribed from an internal promoter (i.e., human $\Delta 133$ ) and contains a a Mouse p53 isoforms Mp53

Mp53AS

(or p44) MA41p53

MA41p53As

MA157p53

MA157p53AS
Human

counterparts

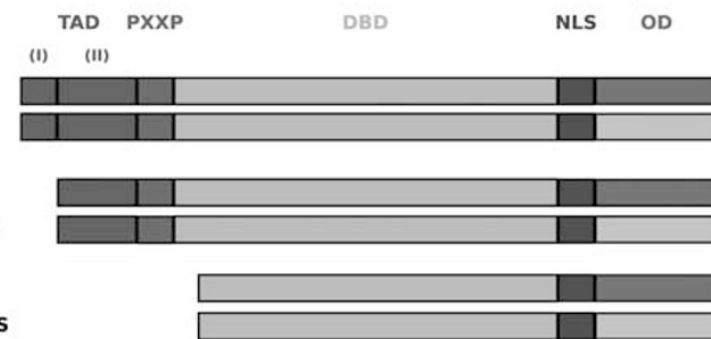
p53
p $53 \beta$
$\triangle 40 \mathrm{p} 53$
$\triangle 40 p 53 \beta$
$\triangle 160 p 53$
$\triangle 160 \mathrm{p} 53 \beta$

Drosophila p53 isoforms

\section{TAD}

Dp53 $\Delta C$

Dp53

DANp53

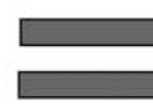

$\square$
DBD

Zebrafish p53 isoforms
DBD

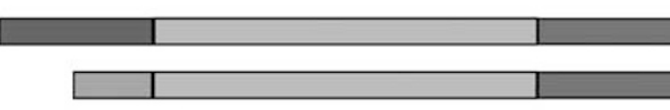

p53

$\triangle 40 / 133 p 53$

Zp53

ZANp53

za113p53

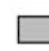

1

b

Buman

Mouse

Drosophila

zebrafish

Buman

Mouse

Drosophila

Zebrafish

Buman

Mouse

Drosophila

Zebrafish

Buman

Mouse

Drosophila

Zebrafish

Buman

Mouse

Drosophila

Zebrafish
ATG40

ATG1

------MEEPQSDPSVEPPLSQETFSDLWKLLPENNVLSPLPSQAMDDLMLSPDDIEQWF ---MTAMEESQSDISLELPLSQETFSGLWKLLPPE---DILPSPECMDDLLLPQDVEEFF MSLHKSASFSLTFNQNTSIVSRSNSRTIFEAFKEFLDFWDIGNEVSAESAVRVSSNGAFN --------MAQNDSQEFAELWEKNIIISIQPPGGGSCWDI INDE------

: : . : : M (intronic) : .

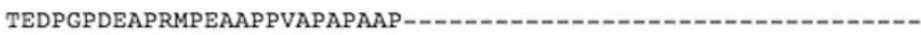

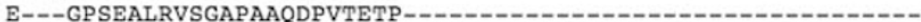
LPQSFGNESNEYAHLATPVDPAYGGNNTNNMMQFTNNLEILANNNSDGNNKINACNKFVC

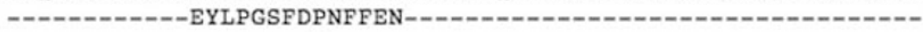
. . *

--TPAAPAPAPSWPLSSSVPSQKTYQG--------------SYGFRLGFLES----------GPVAPAPATPWPLSSFVPSQKTYQG-------------NYGFHLGFLQS-------HKGTDSEDDSTEVDIKEDIPKTVEVSGSELTTEPMAFLQGLNSGNLMQF SQSVLREMML --VLEEQPQPSTLPPTSTVPETSDYPG----------DEGFRLRFPQS-------

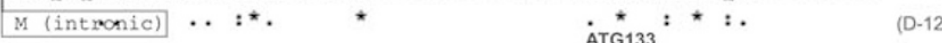

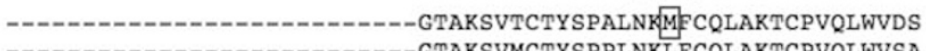
--1-0-1-0.-- GTAKSVMCTY SPPLNKLFCQLAKTCPVQLWVSA QDIQIQANTLPKLENHNIGGYCF SMVLDEPPKSLWMYSIPLNKL Y IRMNKAFNVDVQFKS ---------------GTAKSVTCTYSPDLNKLFCQLAKTCPVQMVVDV (Z-113) ATG160

TPPPGTRVRAMAIYKQSQHMTEVVRRCPHHERCSDSDGLAPPQHLIRVEGNLRVEYL--TPPAGSRVRAMAIYKKSQHMTEVVRRCPHHERCSDGDGLAPPQHLIRVEGNLYPEYL--KMPIQPLNLRVFLCF SNDVSAPVVRCQNHLSVEPLTANNAKMRESLLRSENPNSVYCGNA APPQGSVVRATAIYKKSEHVAEVVRRCPHHERTPDGDNLAPAGHLIRVEGNQRANYR---

Figure 2 p53 isoforms in animal models. (a) Structural organization of p53 isoforms through evolution. Like humans, mouse, Drosophila and zebrafish express a full-length p53 protein, which conserves a TAD (blue), a DNA-binding domain (DBD, orange) and an OD (green). Only the mouse Mp53 protein presents a proline-rich domain (PXXP, purple) and a nuclear localization signal (NLS, green). In addition, all these animals express some p53 isoforms that have the same structural organization as the human p53 isoforms owing to the use of alternative promoters and splicing sites. M, mouse protein; D, Drosophila protein; Z, zebrafish protein; N-terminal p53 isoform identification, $\Delta$ forms; N-terminal p53 isoform denomination, codon number, when initiated ATG occurs in the coding sequence, or N, when initiation occurs in a non-coding sequence; Cterminal p53 isoform identification, AS (alternative splicing, green boxes); grey box, different residues compared with the full-length p53 protein. (b) Localization of translation initiation sites in animal p53 sequences. Red, ATG1 generating the full-length p53 protein; green, the methionine used to produce the homologues to the human $\Delta 40$ p53 forms; blue, the methionine used to produce the homologues to the human $\Delta 133 \mathrm{p} 53$ forms; orange, the methionine used to produce the homologues to the human $\Delta 160 \mathrm{p} 53$ forms. The color reproduction of this figure is available at the Cell Death and Differentiation journal online 
Table 1 Available p53 animal models

\begin{tabular}{|c|c|c|c|}
\hline Species & Name & Modulation of p53 isoforms & Ref. \\
\hline Drosophila & & $\begin{array}{l}\text { Overexpression of } \mathrm{D} \Delta \mathrm{Np} 53 \text { in cells } \\
\text { Overexpression of transgenic } \mathrm{D} \Delta \mathrm{Np} 53 \text { under the control of } \\
\text { tissue-specific promoters } \\
\text { Overexpression of transgenic dominant-negative mutant } \mathrm{D} \Delta \mathrm{Np} 53 \\
\text { Inactivation of } D p 53 \text { locus }\end{array}$ & $\begin{array}{l}45 \\
47,49-53 \\
53,54 \\
49,53\end{array}$ \\
\hline Zebrafish & $\operatorname{Tg}(\Delta 113 p 53: g f p)$ & $\begin{array}{l}\text { Overexpression of transgenic } Z \Delta 113 p 53-G F P \text { morpholino ( } \Delta 113 p 53-M O) \\
\text { Overexpression of ectopic } Z \Delta N p 53\end{array}$ & $\begin{array}{l}61 \\
59\end{array}$ \\
\hline \multirow[t]{2}{*}{ Mouse } & $\mathrm{M} \Delta 41 \mathrm{p} 53 / \mathrm{M} \Delta 41 \mathrm{p} 53 \mathrm{wt} / \mathrm{M} \Delta 41 \mathrm{p} 53$ & Overexpression of transgenic $\mathrm{M} \Delta 41 \mathrm{p} 53$ & 65 \\
\hline & $\begin{array}{l}\mathrm{M} \Delta 122 \mathrm{p} 53 / \mathrm{M} \Delta 122 \mathrm{p} 53 \mathrm{wt} / \mathrm{M} \Delta 122 \mathrm{p} 53 \\
\mathrm{~m} / \mathrm{m} \text { wt/m }\end{array}$ & $\begin{array}{l}\text { Overexpression of transgenic } \mathrm{M} \Delta 122 \mathrm{p} 53 \\
\text { 'm'= deletion of exon } 1-6\end{array}$ & $\begin{array}{l}68 \\
69\end{array}$ \\
\hline
\end{tabular}

truncated TAD followed by a complete DBD and OD (i.e., human $\Delta 40$ ); and Dp53 $\Delta \mathrm{C}$, encoded by a short transcript leading to a putative isoform bearing only the TAD. ${ }^{33,45-47}$ The Drosophila p53 gene is activated by irradiation and exerts broad suppressive effects recapitulating those of the p53-family members, including regulation of apoptosis, aging, autophagy, differentiation and growth. ${ }^{48}$ Historically, D $\Delta$ Np53 was the first form identified and previously termed Dp53; hence most of the functional studies to date have focused on the role of this particular isoform (Table 1).

Studies on the morphogenesis of imaginal discs have highlighted the role of $\mathrm{D} \Delta \mathrm{Np53}$ isoforms in the control of cell death. ${ }^{45,47,49,50}$ In this system, D $\Delta$ Np53 controls apoptosis through the Reaper-Hid-Grim (RHG) cascade. Indeed, irradiated imaginal discs from flies with a mutant Dp53 gene show reduced apoptosis but normal cell-cycle arrest, suggesting a specific regulatory role of the $D p 53$ gene product in apoptosis. ${ }^{45,49}$ So far, the main isoform implicated in apoptosis appears to be $\mathrm{D} \Delta \mathrm{Np} 53$, which directly regulates reaper (rpr) expression. ${ }^{45}$ However, D $\Delta$ Np53 also exerts effects through other pathways. In particular, D $\Delta$ Np53 may activate either apoptotic or non-apoptotic responses in photoreceptor cells depending on the cell differentiation status. ${ }^{50-52}$ Moreover, D $\Delta$ Np53 appears to inhibit cellular differentiation in the retina independently of its apoptotic function. ${ }^{50}$

The Dp53 gene product also exerts important roles in controlling lifespan in a sex- and stage-dependent manner. When overexpressed in adult flies (Table 1), D $\Delta$ Np53 limited lifespan in females and extended it in males. By contrast, when overexpressed during development, D $\Delta$ Np53 exerted a similar dose-dependent effect on longevity in both sexes. ${ }^{53}$ Conversely, inactivation of the Dp53 locus increased lifespan in females but had only minor effects in males. Similar phenotypes were observed in Drosophila expressing dominant-negative Dp53 mutant transgenes or overexpressing $\mathrm{D} \Delta \mathrm{Np53}$, suggesting that $\mathrm{D} \Delta \mathrm{Np53}$ interferes with Dp53 activity. ${ }^{54}$ The role of Drosophila p53 has also been investigated in detail in other cellular functions such as DNA repair or compensatory proliferation. ${ }^{48,55,56}$ However, the specific roles of each Drosophila p53 isoform, Dp53 and $\mathrm{D} \Delta \mathrm{Np} 53$, have not been studied in sufficient detail to understand the exact contributions of each isoform to development, stress responses or longevity.

Danio rerio, the historical model to study p53 isoforms. The zebrafish p53 protein, Zp53, recapitulates the suppressive and pro-apoptotic functions of human p53 upon genotoxic stress. ${ }^{57}$ So far, only $\mathrm{N}$-terminal Zp53 isoforms have been identified (Figure 2): Zp53, corresponding to the human p53 protein; ${ }^{58} \mathrm{Z} \Delta \mathrm{Np} 53$, produced through an alternative splicing of intron-2 and thus similar to the human $\Delta 40 p 53$ forms; ${ }^{59}$ and $Z \Delta 113 p 53$, produced by an internal promoter located within the zebrafish $Z p 53$ gene that is regulated by $\mathrm{Zp} 53$ itself, and thus equivalent to the human $\Delta 133$ p53 isoform. ${ }^{37,38,60}$ In contrast to the human $\triangle 40 p 53$, AUG1 of $Z \Delta N p 53$ is located within the partial intronic sequence retained by alternative splicing. ${ }^{59}$ Thus in $\mathrm{Z} \triangle \mathrm{Np} 53$, the $38 \mathrm{~N}$-terminal residues containing the TAD are replaced by 33 residues derived from the intron-2 sequence.

There is evidence that the $Z \Delta N p 53$ transcript accumulates in response to $\gamma$-ray irradiation. ${ }^{59}$ In addition, ectopic expression of $Z \Delta N p 53$ resulted in a strong developmental phenotype with hypoplasia and malformation of the head, eyes and somites (Table 1). This phenotype is dependent upon the presence of $\mathrm{Zp53}$, the two isoforms forming a protein complex through their ODs. Overexpression of an OD-mutant $Z \Delta N p 53$ or wild-type $Z \Delta N p 53$ in a mutant Zp53 background is phenotypically ineffective. These observations suggest that $\mathrm{Z} \Delta \mathrm{Np53}$ exerts its effects by modulating the activity of Zp53 during zebrafish development.

$\mathrm{Z} \Delta 113 \mathrm{p} 53$ was discovered in a different context (Table 1). Cheng and co-workers found that in zebrafish embryos, loss of the Def gene (Digestive organ Expansion Factor) led to defects in the morphogenesis of digestive organs. In a genome-wide screen, they identified a shorter form of Zp53 whose expression was upregulated in def $^{-1}$ embryos. ${ }^{60}$ Upregulation of $Z \Delta 113 p 53$ correlated with increased expression of p53-target genes involved in cell-cycle progression such as cyclin-G1 and $p 21^{\text {WAF1 }}$, whereas pro-apoptotic genes such as Bax and Reprimo were not activated. Furthermore, $\mathrm{Z} \Delta 113 \mathrm{p} 53$ was found to selectively upregulate the $B C l-2 L$ antiapoptotic gene.$^{61}$ This pattern of effects is consistent with the notion that $Z \Delta 113 p 53$, like $\Delta 133 p 53$, operates as a modulator of p53 in selectively activating defined target genes.

Mouse, the next generation of animal models. Six p53 isoforms have been described in mice, resulting from combination of three $\mathrm{N}$-terminal p53 isoforms with two different C-terminal isoforms (Figure 2). In addition to the full-length mouse Mp53, Rotter and co-workers identified an alternative p53 mRNA retaining part of intron-10 that encodes a shorter isoform with new residues in place of 
the usual OD (Mp53AS), homologous to the human $\mathrm{p} 53 \beta .^{10,62}$ Later, Mowat et al. ${ }^{63}$ isolated $\mathrm{M} \Delta 41 \mathrm{p} 53$, the mouse counterpart of human $\Delta 40$ p53 forms. In addition, Khoury et al. isolated a shorter $\mathrm{N}$-terminal form produced by an internal promoter within the mouse $p 53$ gene, $M \Delta 157 p 53$, equivalent to the human $\Delta 160 p 53$ form. Khoury et al. also showed that $M \Delta 41 p 53$ and $M \Delta 157 p 53$ can be expressed as a C-terminal AS variant. As for the human p53 isoforms, Rotter and co-workers reported that Mp53AS modulates Mp53-mediated apoptosis and Mp53 transcriptional activity in luciferase reporter assays. ${ }^{64}$

In 2004, Maier et al. ${ }^{65}$ described a transgenic mouse overexpressing $M \Delta 41 p 53$ (Table 1). When expressed in a p53-null background, this isoform did not induce any particular phenotype. However, when expressed in a p53-competent background, an increased dosage of $M \Delta 41 \mathrm{p} 53$ led to reduced size, accelerated aging and a shorter lifespan associated with hypo-insulinemia and glucose insufficiency. ${ }^{65-67}$ These effects were attributed to the hyper-activation of the insulinlike growth factor (IGF)-signalling axis by $\mathrm{M} \Delta 41 \mathrm{p} 53$, setting in motion a cascade that clamps unimpeded growth through

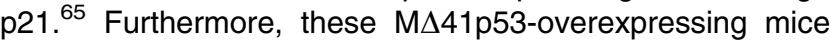
show cognitive decline and synaptic impairment early in life, also attributable to the hyper-activation of the IGF-1-signalling pathway. ${ }^{67}$ These observations are consistent with studies performed in vitro and in the zebrafish model, indicating that $\Delta 40$ p53 isoforms may regulate growth suppression through modulation of p53 activity.

A premature aging phenotype was also reported in a nonphysiological knock-in p53 mouse model expressing a 'M $\Delta 122 \mathrm{p} 53$ ' form, truncated for the first 122 residues (Table 1). ${ }^{68}$ Although $\mathrm{M} \Delta 122 \mathrm{p} 53$ has no physiological equivalent in mouse, it can be considered as an 'intermediate' between the $M \Delta 41 p 53$ and $M \Delta 157 p 53$ isoforms, as it lacks the TAD and part of the DNA-binding domain. During adulthood, transgenic $p 53^{\Delta 122 p 53 / \Delta 122 p 53}$ mice showed premature aging symptoms, such as balding and arthritis, similar to that observed in the $\mathrm{p} 53^{+/ \mathrm{m}}$ mice (deletion exon 1-6).$^{68,69}$ In addition, earlier tumour onset and shortened lifespan were observed in $\mathrm{p} 53^{+/ \Delta 122 \mathrm{p} 53}$ mice as compared with $\mathrm{p} 53^{+/-}$ mice. Overall, these results support the hypothesis that mouse $\mathrm{N}$-terminal p53 forms may operate as dominant oncogenes to promote cell proliferation and inflammation.

The studies summarized above highlight common themes in the 'p53 isoform' field. First, overall patterns of isoform expression are well-conserved throughout evolution. Second, $\mathrm{N}$-terminal isoforms have a major role as regulators of physiological processes related to development, aging, lifespan and, possibly, carcinogenesis. In this respect, two key mechanisms are emerging. The $\Delta 40 p 53$ form exerts regulatory effects on signalling cascades controlled by p53, perhaps through direct interaction between the two isoforms. Conversely, isoforms corresponding to $\Delta 133 p 53 \alpha$ modulate cell response by regulating gene expression in a p53-dependent and -independent manner. It should, however, be remembered that our current view of isoform activities remains fragmentary and that further studies are needed to better understand their roles and underlying mechanisms.

\section{p53 Isoforms and Human Cancers}

Genetic polymorphisms: effects on p53 isoform expression. The TP53 gene is highly polymorphic, with over $80 \%$ of known single-nucleotide polymorphisms (SNPs) located within introns or non-coding $5^{\prime}$ and $3^{\prime}$ sequences. ${ }^{70}$ Hainaut and co-workers showed that G-quadruplex structures, formed in intron-3 and regulating intron-2 splicing (and thus $\Delta 40$ form expression), overlap a common polymorphism, TP53 PIN3, which consists of a 16-bp duplication (A1, non-duplicated allele; A2, duplicated allele). ${ }^{30,71}$ This polymorphism may therefore modulate the structure and/or the stability of the G-quadruplexes, and affect $\Delta 40$ p53 expression (Table 2). Consistent with a functional effect, TP53 PIN3 has recently been identified as a strong genetic modifier of germline TP53 mutations. Indeed, carriers bearing A1A1 genotypes developed their first cancer on average 20 years earlier than carriers with an A1A2 genotype. ${ }^{72}$ This effect was detected in a Brazilian cohort, in which the p.R337H mutation of partial penetrance is very common owing to a widespread founder effect. ${ }^{5}$ It remains to be demonstrated whether a similar effect is observed in carriers of other TP53 mutation types.

Landi and co-workers have identified 11 different haplotypes defined by eight SNPs in a region from exon-3 to intron-4, overlapping part of the internal P2 promoter regulating $\Delta 133 p 53$ and $\Delta 160 p 53$ expression. ${ }^{73}$ Using these different haplotypes as promoters to drive luciferase expression, they found significant differences in basal promoter activity that were confirmed by analysing endogenous

Table 2 Role of p53 isoforms in human cancers

\begin{tabular}{|c|c|c|c|}
\hline Cancer & Isoform & Observation & Ref. \\
\hline Breast cancer & $\begin{array}{l}\Delta 133 p 53 \alpha \\
\text { p53 } \beta \\
\text { p53 } \gamma\end{array}$ & $\begin{array}{l}\text { Overexpression } \\
\text { Loss of expression } \\
\text { Loss of expression } \\
\text { Prognosis of mutp53/p53 } \gamma \text { as good as wtp53 }\end{array}$ & $\begin{array}{c}33 \\
33,78 \\
33,79\end{array}$ \\
\hline Acute myeloid leukaemia & $\mathrm{p} 53 \beta / \gamma$ & Increased expression in response to chemotherapy & 78 \\
\hline \multirow[t]{2}{*}{ Ovarian cancer } & $\mathrm{p} 53 \beta$ & $\begin{array}{l}\text { Serous and poorly differentiated tumours/worse recurrence-free } \\
\text { and overall survival }\end{array}$ & 77 \\
\hline & $\Delta 40 p 53$ & Improved recurrence-free survival & 74 \\
\hline Li-Fraumeni syndrome & $\Delta 40 p 53$ & $\begin{array}{l}\text { G-quadruplex structure overlapping TP53 PIN3/early age at first cancer } \\
\text { diagnosis in patients carrying A1A1 compared with patients carrying A1A2 }\end{array}$ & 72 \\
\hline
\end{tabular}


$\Delta 133 p 53$ expression in samples of normal colonic mucosa. Furthermore, in silico and in vitro DNA binding analyses suggested that an SNP in intron-4 (rs179287; C > T) affects proteins binding within the $\mathrm{P} 2$ promoter. ${ }^{73}$ These results suggest that genetic polymorphisms may modulate basal $\Delta 133 p 53$ expression. Whether these effects influence cancer risk remains to be demonstrated.

Mutations affecting the production of isoforms. Given the role of $\mathrm{N}$-terminal p53 forms as inhibitors of p53 transactivation, it can be expected that overexpression of certain isoforms may represent an alternative to a mutation in TP53 for inactivating p53 in cancers, as observed in some small clinical studies. ${ }^{33,74,75}$ Analysis of the IARC TP53 database identified 1019 somatic cancer mutations that are predicted to disrupt the p53 coding sequence in the $\mathrm{N}$-terminal region while leaving intact the sequence encoding at least $\Delta 160 p 53$. This represents $3.65 \%$ of all somatic mutations reported to date (version R15; wwwp53.iarc.fr). ${ }^{70}$ Therefore, rare and/or silent mutations in TP53 may differentially affect the expression of p53 isoforms, as observed recently for mutations present in the IRES regulating the relative expression of $p 53$ and $\Delta 40 p 53$ forms encoded by the full-length FSp53 mRNA. ${ }^{76}$ With respect to C-terminal isoforms, Hofstetter et al. $^{77}$ have used an yeastbase functional assay and RT-PCR to show that mutations at splice sites of TP53 intron-9 can lead to aberrant expression of $\mathrm{p} 53 \beta$ mRNA in primary cultures of ovarian cancer cells. By contrast, somatic mutations at splice sites of introns 6 and 9 were found to generate spliced mutant p53 mRNAs (p53\%, p53 $\delta$ and $\mathrm{p} 53 \varepsilon$ ), distinct from the physiological isoforms. However, the role of such mutant isoforms in carcinogenesis is not known.

Isoform expression and cancer outcomes. There is emerging evidence that p53 isoform expression is deregulated in human cancers (Table 2). In a study of 245 primary ovarian cancers, Hofstetter et al. ${ }^{77}$ observed that expression of $\mathrm{p} 53 \beta$ was associated with serous and poorly differentiated cancers, and, when expressed together with the functional p53 protein, it was correlated with poor recurrence-free and overall survival. By contrast, tumours expressing functional p53 and $\Delta 40$ p53 showed improved recurrence-free survival of patients compared with tumours expressing no $\Delta 40 p 53$. Studies of patients with acute myeloid leukemia have shown that elevated expression of p53 $\beta$ and/or p53 $\gamma$ in blood cells was correlated with improved responses to chemotherapy, ${ }^{78}$ which may predict decreased chemoresistance and improved overall survival. An effect of isoform expression has also been observed on breast cancer prognosis. In a cohort of 127 breast cancer patients, Thompson and co-workers reported that patients whose tumours expressed both mutant p53 and p53 $\gamma$ mRNAs had a prognosis as good as patients whose tumours expressed a wild-type p53, suggesting that the expression of $p 53 \gamma$ may abrogate the poor prognosis commonly associated with TP53 mutations. ${ }^{79}$

\section{Conclusion}

The field of 'p53 isoforms' is still in its infancy, but the increasing number of genetic, biochemical and clinical studies have clearly established that p53 isoforms are fundamental and important components of the p53 pathway. Data obtained from animal and cellular models indicate that p53 isoforms regulate the cell fate in response to developmental defects and cell damages by differentially regulating gene expression, both in a p53-dependent and -independent manner. Furthermore, the current data suggest that p53 isoforms have roles in all biological activities regulated by p53 (Figure 3). Therefore, one can reasonably expect that the characterization of the biochemical and biological activities of p53 isoforms will impact on the fields of cancer, embryo development and aging.

Future experiments will be needed to gain further insight into how p53 isoforms modulate the different biological

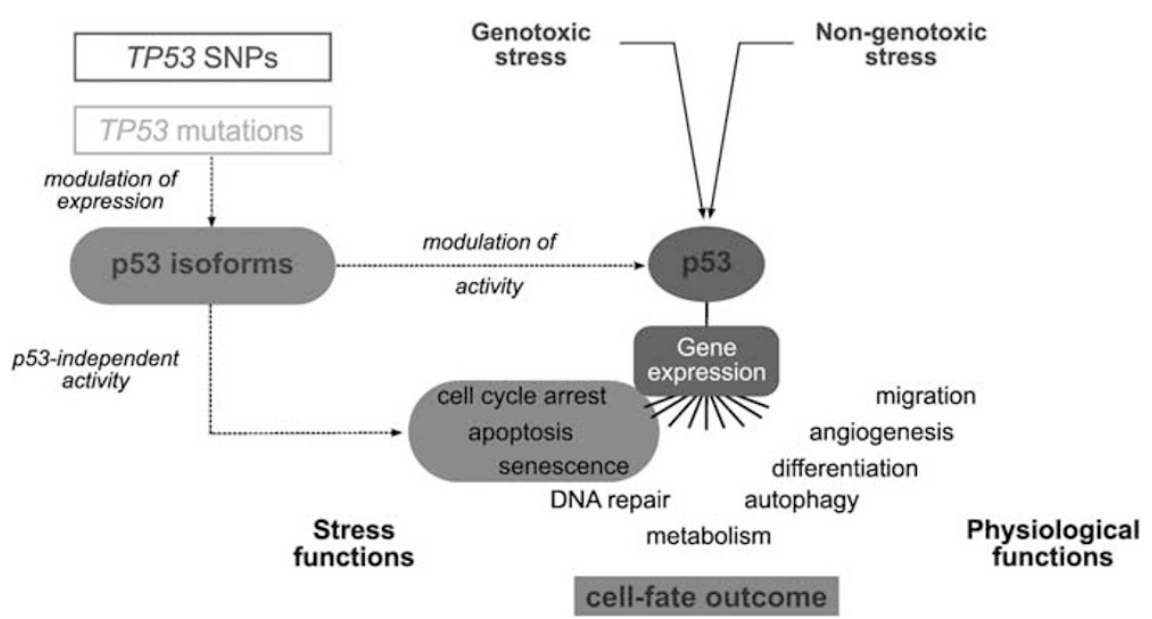

Figure 3 p53 isoforms in the p53 network. p53 integrates the different stress signals to adapt cell fate to the intensity and the nature of stress by regulating several biological functions to maintain genomic and cellular integrity. In addition, p53 controls physiological functions under basal conditions. Recent data suggest that p53 isoforms modulate p53-mediated cell fate outcome and may thus be key components of the p53-mediated decision not only in response to stress but also under basal conditions. It has also been reported that p53 isoforms have p53-independent activities and directly regulate cell-cycle arrest and apoptosis. In addition, genetic alterations, such as TP53 SNPs and TP53 mutations, affect the expression of p53 isoforms, which may result in tumorigenesis 
activities. However, based on our current understanding of the p63, p73 and p53 isoforms, we have come to realize that the p53 pathway should no longer be considered as regulated by only the p53 protein, but by a set of p63, p73 and p53 isoforms that interplay with each other in regulating physiological functions. Further progress on this front will require the development of robust and standardized tools for the identification and quantification of p53, p63 and p73 protein isoform expression in experimental systems as well as in human tissues.

\section{Conflict of Interest}

The authors declare no conflict of interest.

Acknowledgements. We thank all the speakers for their participation in the 1st International p53 Isoforms Meeting, and Michelle Wrisez for logistic help. The meeting was funded by IARC, the College of Medicine of the University of Dundee and the Laboratory of Molecular Biology of the Cell (UMR5239, CNRS at ENS de Lyon). Links: IARC TP53 database, http://www-p53.iarc.fr/; abstracts of the International p53 Isoforms Meeting, http://www-p53.iarc.fr/ Download/Book_p53lsoforms2010_Final.pdf. VM is supported by a fellowship from the Breast Cancer Campaign; M-LD-D and BM by CNRS ATIP, Fondation pour la Recherche Médicale (FRM) and La Ligue contre le cancer; CS by a PhD grant from the French Ministry of Research; JH by INSERM and Institut Curie; $\mathrm{PH}$ and JH by I'Insititut National contre le Cancer (INCa, France; Projet Libre 2009, 2009-192); and J-CB by Cancer Research UK.

\section{Facts}

- At least nine different mRNAs are expressed from the TP53 gene encoding 12 distinct protein isoforms through the use of alternative promoters, splicing sites and internal initiation sites of translation.

- p53 isoforms regulate cell fate in response to developmental defects and cell damage by differentially regulating gene expression, both in a p53-dependent and -independent manner.

- The $\mathrm{N}$-terminal p53 isoforms, $\Delta 40$ and $\Delta 133$, are not strict dominant-negative inhibitors of p53's suppressive function as they have p53-independent activities.

- The expression patterns and biological activities of p53 isoform are conserved through evolution (human, mouse, zebrafish and Drosophila).

- TP53SNPs and mutations alter the pattern of p53 isoform expression.

- p53 isoform expression is deregulated in human cancers and is associated with clinical prognosis.

\section{Open Questions}

- Development of standardized and robust tools is needed to systematically identify and quantify p53 isoforms in human and animal models.

- Characterization of the biochemical activities of p53 isoforms as well as their molecular targets.

- Is each p53 isoform specialized in a particular biological response? Have they redundant activities?

- Evaluation of the interplay between p53-, p63- and p73-family isoforms.

- Study the potential use of p53 isoform expression in human cancers as biomarkers and as therapeutic targets.
1. Levine AJ, Oren M. The first 30 years of p53: growing ever more complex. Nat Rev Cancer 2009; 9: 749-758.

2. Levine AJ, Tomasini R, McKeon FD, Mak TW, Melino G. The p53 family: guardians of maternal reproduction. Nat Rev Mol Cell Biol 2011; 12: 259-265.

3. Meek DW, Anderson CW. Posttranslational modification of p53: cooperative integrators of function. Cold Spring Harb Perspect Biol 2009; 1: a000950.

4. Vilborg A, Wilhelm MT, Wiman KG. Regulation of tumor suppressor p53 at the RNA level. J Mol Med 2010; 88: 645-652.

5. Garritano S, Gemignani F, Palmero El, Olivier M, Martel-Planche G, Le Calvez-Kelm F et al. Detailed haplotype analysis at the TP53 locus in p.R337H mutation carriers in the population of Southern Brazil: evidence for a founder effect. Hum Mutat 2010; 31: $143-150$

6. Khoury MP, Bourdon JC. The isoforms of the p53 protein. Cold Spring Harb Perspect Biol 2010; 2: a000927.

7. Marcel V, Hainaut P. p53 isoforms - a conspiracy to kidnap p53 tumor suppressor activity? Cell Mol Life Sci 2009; 66: 391-406

8. Flaman JM, Waridel F, Estreicher A, Vannier A, Limacher JM, Gilbert D et al. The human tumour suppressor gene p53 is alternatively spliced in normal cells. Oncogene 1996; 12: 813-818.

9. Matlashewski G, Lamb P, Pim D, Peacock J, Crawford L, Benchimol S. Isolation and characterization of a human p53 cDNA clone: expression of the human p53 gene. EMBO J 1984; 3: 3257-3262.

10. Wolf $\mathrm{D}$, Harris N, Goldfinger N, Rotter V. Isolation of a full-length mouse cDNA clone coding for an immunologically distinct p53 molecule. Mol Cell Biol 1985; 5: 127-132.

11. Marcel V, Olivier M, Mollereau B, Hainaut P, Bourdon JC. First International p53 Isoforms Meeting: 'p53 isoforms through evolution: from identification to biological function'. Cell Death Differ 2011; 18: 563-564.

12. Melino G. p63 is a suppressor of tumorigenesis and metastasis interacting with mutant p53. Cell Death Differ 2011; 18: 1487-1499.

13. Murray-Zmijewski F, Lane DP, Bourdon JC. p53/p63/p73 isoforms: an orchestra of isoforms to harmonise cell differentiation and response to stress. Cell Death Differ 2006; 13: $962-972$

14. Kaghad M, Bonnet $\mathrm{H}$, Yang $\mathrm{A}$, Creancier L, Biscan JC, Valent $\mathrm{A}$ et al. Monoallelically expressed gene related to $\mathrm{p} 53$ at $1 \mathrm{p} 36$, a region frequently deleted in neuroblastoma and other human cancers. Cell 1997; 90: 809-819.

15. Yang A, Walker N, Bronson R, Kaghad M, Oosterwegel M, Bonnin J et al. p73-deficient mice have neurological, pheromonal and inflammatory defects but lack spontaneous tumours. Nature 2000; 404: 99-103.

16. Yang A, Kaghad M, Wang Y, Gillett E, Fleming MD, Dotsch V et al. p63, a p53 homolog at $3 q 27-29$, encodes multiple products with transactivating, death-inducing, and dominantnegative activities. Mol Cell 1998; 2: 305-316.

17. Mills AA, Zheng B, Wang XJ, Vogel H, Roop DR, Bradley A. p63 is a p53 homologue required for limb and epidermal morphogenesis. Nature 1999; 398: 708-713.

18. Senoo M, Pinto F, Crum CP, McKeon F. p63 is essential for the proliferative potential of stem cells in stratified epithelia. Cell 2007: 129: 523-536.

19. Su X, Chakravarti D, Cho MS, Liu L, Gi YJ, Lin YL et al. TAp63 suppresses metastasis through coordinate regulation of Dicer and miRNAs. Nature 2010; 467: 986-990.

20. Su X, Paris M, Gi YJ, Tsai KY, Cho MS, Lin YL et al. TAp63 prevents premature aging by promoting adult stem cell maintenance. Cell Stem Cell 2009; 5: 64-75.

21. Medawar A, Virolle T, Rostagno P, de la Forest-Divonne S, Gambaro K, Rouleau M et al. DeltaNp63 is essential for epidermal commitment of embryonic stem cells. PLoS One 2008; 3: e3441.

22. Shalom-Feuerstein R, Lena AM, Zhou H, De La Forest Divonne S, Van Bokhoven H, Candi $\mathrm{E}$ et al. DeltaNp63 is an ectodermal gatekeeper of epidermal morphogenesis. Cell Death Differ 2011; 18: 887-896.

23. Keyes WM, Pecoraro M, Aranda V, Vernersson-Lindahl E, Li W, Vogel H et al. DeltaNp63alpha is an oncogene that targets chromatin remodeler Lsh to drive skin stem cell proliferation and tumorigenesis. Cell Stem Cell 2011; 8: 164-176.

24. Guo X, Keyes WM, Papazoglu C, Zuber J, Li W, Lowe SW et al. TAp63 induces senescence and suppresses tumorigenesis in vivo. Nat Cell Biol 2009; 11: 1451-1457.

25. Wilhelm MT, Rufini A, Wetzel MK, Tsuchihara K, Inoue S, Tomasini R et al. Isoform-specific p73 knockout mice reveal a novel role for delta Np73 in the DNA damage response pathway. Genes Dev 2010; 24: 549-560.

26. Tomasini R, Tsuchihara K, Wilhelm M, Fujitani M, Rufini A, Cheung CC et al. TAp73 knockout shows genomic instability with infertility and tumor suppressor functions. Genes Dev 2008; 22: 2677-2691.

27. Matlashewski G, Pim D, Banks L, Crawford L. Alternative splicing of human p53 transcripts. Oncogene Res 1987; 1: 77-85.

28. Ghosh A, Stewart D, Matlashewski G. Regulation of human p53 activity and cell localization by alternative splicing. Mol Cell Biol 2004; 24: 7987-7997.

29. Courtois S, Verhaegh G, North S, Luciani MG, Lassus P, Hibner U et al. DeltaN-p53, a natural isoform of p53 lacking the first transactivation domain, counteracts growth suppression by wild-type p53. Oncogene 2002; 21: 6722-6728.

30. Marcel V, Tran PL, Sagne C, Martel-Planche G, Vaslin L, Teulade-Fichou MP et al. G-quadruplex structures in TP53 intron 3: role in alternative splicing and in production of p53 mRNA isoforms. Carcinogenesis 2011; 32: 271-278. 
31. Candeias MM, Powell DJ, Roubalova E, Apcher S, Bourougaa K, Vojtesek B et al. Expression of $p 53$ and p53/47 are controlled by alternative mechanisms of messenger RNA translation initiation. Oncogene 2006; 25: 6936-6947.

32. Ray PS, Grover R, Das S. Two internal ribosome entry sites mediate the translation of p53 isoforms. EMBO Rep 2006; 7: 404-410.

33. Bourdon JC, Fernandes K, Murray-Zmijewski F, Liu G, Diot A, Xirodimas DP et al. p53 isoforms can regulate p53 transcriptional activity. Genes Dev 2005; 19: 2122-2137.

34. Reisman D, Balint E, Loging WT, Rotter V, Almon E. A novel transcript encoded within the $10-\mathrm{kb}$ first intron of the human p53 tumor suppressor gene (D17S2179E) is induced during differentiation of myeloid leukemia cells. Genomics 1996; 38: 364-370.

35. Reisman D, Greenberg M, Rotter V. Human p53 oncogene contains one promoter upstream of exon 1 and a second, stronger promoter within intron 1. Proc Natl Acad Sci USA 1988; 85: 5146-5150.

36. Marcel V, Perrier S, Aoubala M, Ageorges S, Groves MJ, Diot A et al. Delta160p53 is a novel N-terminal p53 isoform encoded by Delta133p53 transcript. FEBS Lett 2010; 584 4463-4468.

37. Aoubala M, Murray-Zmijewski F, Khoury MP, Fernandes K, Perrier S, Bernard $\mathrm{H}$ et al. p53 directly transactivates Delta133p53alpha, regulating cell fate outcome in response to DNA damage. Cell Death Differ 2011; 18: 248-258.

38. Marcel V, Vijayakumar V, Fernandez-Cuesta L, Hafsi H, Sagne C, Hautefeuille A et al. p53 regulates the transcription of its Delta133p53 isoform through specific response elements contained within the TP53 P2 internal promoter. Oncogene 2010; 29: 2691-2700.

39. Fuller-Pace FV, Ali S. The DEAD box RNA helicases p68 (Ddx5) and p72 (Ddx17): novel transcriptional co-regulators. Biochem Soc Trans 2008; 36 (Part 4): 609-612.

40. Graupner V, Schulze-Osthoff K, Essmann F, Janicke RU. Functional characterization of p53beta and p53gamma, two isoforms of the tumor suppressor p53. Cell Cycle 2009; 8: 1238-1248.

41. Yin Y, Stephen CW, Luciani MG, Fahraeus R. p53 stability and activity is regulated by Mdm2-mediated induction of alternative p53 translation products. Nat Cell Biol 2002; 4: 462-467.

42. Brady CA, Jiang D, Mello SS, Johnson TM, Jarvis LA, Kozak MM et al. Distinct p53 transcriptional programs dictate acute DNA-damage responses and tumor suppression. Cell 2011; 145: 571-583.

43. Ohki R, Kawase T, Ohta T, Ichikawa H, Taya Y. Dissecting functional roles of p53 $\mathrm{N}$-terminal transactivation domains by microarray expression analysis. Cancer Sci 2007; 98: 189-200.

44. Fujita K, Mondal AM, Horikawa I, Nguyen GH, Kumamoto K, Sohn JJ et al. p53 isoforms Delta133p53 and p53beta are endogenous regulators of replicative cellular senescence. Nat Cell Biol 2009; 11: 1135-1142.

45. Brodsky MH, Nordstrom W, Tsang G, Kwan E, Rubin GM, Abrams JM. Drosophila p53 binds a damage response element at the reaper locus. Cell 2000; 101: 103-113.

46. Jin S, Martinek S, Joo WS, Wortman JR, Mirkovic N, Sali A et al. Identification and characterization of a p53 homologue in Drosophila melanogaster. Proc Natl Acad Sci USA 2000; 97: 7301-7306.

47. Ollmann M, Young LM, Di Como CJ, Karim F, Belvin M, Robertson S et al. Drosophila p53 is a structural and functional homolog of the tumor suppressor p53. Cell 2000; 101: 91-101.

48. Rutkowski R, Hofmann K, Gartner A. Phylogeny and function of the invertebrate p53 superfamily. Cold Spring Harb Perspect Biol 2010; 2: a001131.

49. Brodsky MH, Weinert BT, Tsang G, Rong YS, McGinnis NM, Golic KG et al. Drosophila melanogaster MNK/Chk2 and p53 regulate multiple DNA repair and apoptotic pathways following DNA damage. Mol Cell Biol 2004; 24: 1219-1231.

50. Fan Y, Lee TV, Xu D, Chen Z, Lamblin AF, Steller H et al. Dual roles of Drosophila p53 in cell death and cell differentiation. Cell Death Differ 2010; 17: 912-921.

51. Mendes CS, Levet C, Chatelain G, Dourlen P, Fouillet A, Dichtel-Danjoy ML et al. ER stress protects from retinal degeneration. EMBO J 2009; 28: 1296-1307.

52. Peterson C, Carney GE, Taylor BJ, White K. reaper is required for neuroblast apoptosis during Drosophila development. Development 2002; 129: 1467-1476.

53. Waskar M, Landis GN, Shen J, Curtis C, Tozer K, Abdueva D et al. Drosophila melanogaster p53 has developmental stage-specific and sex-specific effects on adult life span indicative of sexual antagonistic pleiotropy. Aging (Albany NY) 2009; 1: 903-936.

54. Bauer JH, Poon PC, Glatt-Deeley H, Abrams JM, Helfand SL. Neuronal expression of p53 dominant-negative proteins in adult Drosophila melanogaster extends life span. Curr Biol 2005; 15: 2063-2068.
55. Lu B. Recent advances in using Drosophila to model neurodegenerative diseases. Apoptosis 2009; 14: 1008-1020.

56. Martin FA, Perez-Garijo A, Morata G. Apoptosis in Drosophila: compensatory proliferation and undead cells. Int J Dev Biol 2009; 53: 1341-1347.

57. Storer NY, Zon LI. Zebrafish models of p53 functions. Cold Spring Harb Perspect Biol 2010; 2: a001123.

58. Cheng R, Ford BL, O'Neal PE, Mathews CZ, Bradford CS, Thongtan T et al. Zebrafish (Danio rerio) p53 tumor suppressor gene: cDNA sequence and expression during embryogenesis. Mol Mar Biol Biotechnol 1997; 6: 88-97.

59. Davidson WR, Kari C, Ren Q, Daroczi B, Dicker AP, Rodeck U. Differential regulation of p53 function by the $\mathrm{N}$-terminal DeltaNp53 and Delta113p53 isoforms in zebrafish embryos. BMC Dev Biol 2010; 10: 102.

60. Chen J, Ruan H, Ng SM, Gao C, Soo HM, Wu W et al. Loss of function of def selectively upregulates Delta113p53 expression to arrest expansion growth of digestive organs in zebrafish. Genes Dev 2005; 19: 2900-2911.

61. Chen J, Ng SM, Chang C, Zhang Z, Bourdon JC, Lane DP et al. p53 isoform delta113p53 is a p53 target gene that antagonizes p53 apoptotic activity via BclXL activation in zebrafish. Genes Dev 2009; 23: 278-290.

62. Arai N, Nomura D, Yokota K, Wolf D, Brill E, Shohat $\mathrm{O}$ et al. Immunologically distinct p53 molecules generated by alternative splicing. Mol Cell Biol 1986; 6: 3232-3239.

63. Mowat M, Cheng A, Kimura N, Bernstein A, Benchimol S. Rearrangements of the cellular p53 gene in erythroleukaemic cells transformed by Friend virus. Nature 1985; 314 633-636.

64. Almog N, Goldfinger N, Rotter V. p53-dependent apoptosis is regulated by a C-terminally alternatively spliced form of murine p53. Oncogene 2000; 19: 3395-3403.

65. Maier B, Gluba W, Bernier B, Turner T, Mohammad K, Guise T et al. Modulation of mammalian life span by the short isoform of p53. Genes Dev 2004; 18: 306-319.

66. Hinault C, Kawamori D, Liew CW, Maier B, Hu J, Keller SR et al. \{Delta\}40 isoform of p53 controls \{beta\}-cell proliferation and glucose homeostasis in mice. Diabetes 2011; 60: 1210-1222.

67. Pehar M, O'Riordan KJ, Burns-Cusato M, Andrzejewski ME, del Alcazar CG, Burger C et al. Altered longevity-assurance activity of p53:p44 in the mouse causes memory loss, neurodegeneration and premature death. Aging Cell 2010; 9: 174-190.

68. Slatter TL, Hung N, Campbell H, Rubio C, Mehta R, Renshaw $\mathrm{P}$ et al. Hyperproliferation, cancer, and inflammation in mice expressing a \{Delta\}133p53-like isoform. Blood 2011; 117: $5166-5177$.

69. Tyner SD, Venkatachalam S, Choi J, Jones S, Ghebranious N, Igelmann $\mathrm{H}$ et al. p53 mutant mice that display early ageing-associated phenotypes. Nature 2002; 415: 45-53.

70. Petitjean $A$, Mathe $E$, Kato $S$, Ishioka $C$, Tavtigian SV, Hainaut $P$ et al. Impact of mutan p53 functional properties on TP53 mutation patterns and tumor phenotype: lessons from recent developments in the IARC TP53 database. Hum Mutat 2007; 28: 622-629.

71. Lazar V, Hazard F, Bertin F, Janin N, Bellet D, Bressac B. Simple sequence repeat polymorphism within the p53 gene. Oncogene 1993; 8: 1703-1705.

72. Marcel V, Palmero El, Falagan-Lotsch P, Martel-Planche G, Ashton-Prolla P, Olivier M et al. TP53 PIN3 and MDM2 SNP309 polymorphisms as genetic modifiers in the Li-Fraumeni syndrome: impact on age at first diagnosis. J Med Genet 2009; 46: 766-772.

73. Bellini I, Pitto L, Marini MG, Porcu L, Moi P, Garritano S et al. DeltaN133p53 expression levels in relation to haplotypes of the TP53 internal promoter region. Hum Mutat 2010; 31: 456-465.

74. Avery-Kiejda KA, Zhang XD, Adams LJ, Scott RJ, Vojtesek B, Lane DP et al. Small molecular weight variants of p53 are expressed in human melanoma cells and are induced by the DNA-damaging agent cisplatin. Clin Cancer Res 2008; 14: 1659-1668.

75. Machado-Silva A, Perrier S, Bourdon JC. p53 family members in cancer diagnosis and treatment. Semin Cancer Biol 2010; 20: 57-62.

76. Grover R, Sharathchandra A, Ponnuswamy A, Khan D, Das S. Effect of mutations on the p53 IRES RNA structure: implications for de-regulation of the synthesis of p53 isoforms. RNA Biol 2011; 8: 132-142.

77. Hofstetter G, Berger A, Fiegl H, Slade N, Zoric A, Holzer B et al. Alternative splicing of p53 and p73: the novel p53 splice variant p53delta is an independent prognostic marker in ovarian cancer. Oncogene 2010; 29: 1997-2004.

78. Anensen N, Oyan AM, Bourdon JC, Kalland KH, Bruserud O, Gjertsen BT. A distinct p53 protein isoform signature reflects the onset of induction chemotherapy for acute myeloid leukemia. Clin Cancer Res 2006; 12: 3985-3992.

79. Bourdon JC, Khoury MP, Diot A, Baker L, Fernandes K, Aoubala M et al. p53 mutant breast cancer patients expressing p53gamma have as good a prognosis as wild-type p53 breast cancer patients. Breast Cancer Res 2011; 13: R7. 